\title{
PREVALÊNCIA DE STAPHYLOCOCCUS AUREUS METICILINA RESISTENTE EM PROFISSIONAIS DE ENFERMAGEM
}

\section{Carla Juliana Camilo}

Discente do curso de Farmácia do Centro Universitário da Fundação Assis Gurgacz (FAG) e discente de especialização em Análises Clínicas e Toxicológicas, Brasil.

\section{Leyde Daiane de Peder}

Docente no curso de Farmácia do Centro Universitário da Fundação Assis Gurgacz (FAG), Cascavel (PR), Brasil.

E-mail: leydepeder@yahoo.com.br

\section{Claudinei Mesquita da Silva}

Docente no curso de Farmácia do Centro Universitário da Fundação Assis Gurgacz (FAG), Cascavel (PR), Brasil.
RESUMO: O Staphylococcus aureus é um dos patógenos de maior prevalência em Infecções Relacionadas à Assistência à Saúde devido à sua capacidade de sofrer resistência. A disseminação por profissionais de saúde tem sido apontada como predisponente para o aumento desse microrganismo. O objetivo deste estudo foi avaliar a prevalência de $S$. aureus nas mãos e mucosa nasal de profissionais de Enfermagem de um hospital de Cascavel (PR). As amostras coletadas através de swab foram semeadas em ágar manitol salgado 7,5\%. As colônias sugestivas foram submetidas ao Gram e testes bioquímicos da Catalase, DNase e Coagulase. A partir do isolamento, realizou-se teste de suscetibilidade antimicrobiana. A taxa de colonização foi de $56 \%$, com $92 \%$ de MSSA e 8\% de MRSA. Estes dados ressaltam a importância de investigações da prevalência e resistência de $S$. aureus e a necessidade de estudos de vigilância quanto à disseminação de microrganismos em nível hospitalar.

PALAVRAS-CHAVE: Infecção hospitalar; Profissional de saúde; Staphylococcus aureus.

\section{PREVALENCE OF STAPHYLOCOCCUS AUREUS RESISTANT TO METICILLIN IN NURSING PERSONNEL}

ABSTRACT: Staphylococcus aureus is a highly prevalent pathogen in infections related to health assistance due to its resistance. Its dissemination by health professionals has been indicated as a predisponent factor of the micro-organism. Current study evaluates the prevalence of $S$. aureus on the hands and in the nasal mucus of nursing professionals in a hospital in Cascavel PR Brazil. Samples were collected with swabs and seeded in salt mannitol agar 7.5\%. Suggestive colonies underwent gram test and the Catalase, DNase and Coagulase biochemical tests. When isolated, the antimicrobial susceptibility test was performed. Colonization rate reached 56\%, with 92\% MSSA and $8 \%$ MRSA. Data underscore the importance of investigations on the prevalence and resistance of $S$. aureus and the need of vigilance studies on the dissemination of the microorganisms at hospital level.

KEY WORDS: Health professional; Staphylococcus aureus; Hospital infection.

\section{INTRODUÇÃO}

As Infecções Associadas aos Cuidados em Saúde (IACS), termo anteriormente conhecido como Infecções Relacionadas à Assistência 
à Saúde (IRAS), representam um importante problema de saúde pública e de risco à saúde dos usuários que se submetem a procedimentos terapêuticos em hospitais (SILVA; PADOVEZE, 2012). Dessa forma, infecções por bactérias em hospitais tornam-se um desafio e uma prioridade para as instituições de saúde, devido às reduzidas opções terapêuticas para o tratamento dos casos, às complicações relacionadas à assistência clínica e a diminuição da qualidade de vida para o paciente (OLIVEIRA et al., 2012).

Neste contexto, o Staphylococcus aureus, encontrado na pele e nas fossas nasais de pessoas saudáveis, tem demonstrado ser um dos patógenos de maior prevalência em infecções de origem hospitalar. Cerca de $50 \%$ a $87 \%$ das infecçóes relacionadas à assistência à saúde têm como agente responsável o $S$. aureus, desses em 16\% a 43\% dos casos os pacientes evoluem para óbito (BASTOS et al., 2013; MASUNARI; TAVARES, 2007).

Por ser um patógeno com capacidade de desenvolver resistência, atualmente o Staphylococcus aureus é considerado um dos microrganismos mais importantes no contexto das IACS, uma vez que dentre as infecções hospitalares, a incidência de Staphylococcus aureus meticilina (oxacilina) resistente (MRSA) está aumentando progressivamente (BALDUINO, 2008; CORREAL et al., 2013). A meticilina e sua análoga, a oxacilina, são antimicrobianos rotineiramente utilizados em hospitais para o tratamento de infecções causadas por S. aureus (ATIQUE et al., 2012), logo, o Staphylococcus aureus demonstra que a sua virulência e capacidade de adquirir resistência aos agentes antimicrobianos resulta em um problema sério em todo o mundo (CARVALHO et al., 2009).

Dentre os mecanismos que favorecem o aparecimento de cepas responsáveis por infecções hospitalares, está o mecanismo de transmissão de bactérias por profissionais da área da saúde. Estes, uma vez colonizados por microrganismos, podem atuar como vetores, direta ou indiretamente, e podem ainda ser responsáveis principalmente pela transmissão de estirpes patogênicas a pacientes suscetíveis (CARVALHO et al., 2009; FELIX; MIYADAHIRA, 2009). Ademais, a transmissão de microrganismos multirresistentes pode ser dada pelo contato de paciente para paciente ou mais frequentemente por profissionais de saúde. Esses profissionais podem ser portadores de $S$. aureus em sua cavidade nasal e este pode ser transferido para as mãos e pele, vindo a contaminar qualquer superfície que entre em contato com o portador, o que pode desencadear o desenvolvimento de estirpes de Staphylococcus aureus resistentes à meticilina (MRSA) (BRASIL, 2013; CASTRO et al., 2010; XAVIER et al., 2007). Logo, fatores como disseminação de microrganismos por profissionais de saúde têm sido apontados como predisponentes para o aumento na incidência de $S$. aureus (BRASIL, 2013).

Tendo em vista que as infecções por $S$. aureus ocorrem frequentemente em pessoas colonizadas com o microrganismo, sendo os carreadores de longo tempo, este se torna o fator de risco mais fortemente associado à infecção subsequente (MOURA et al., 2011). Em função desse conhecimento, torna-se fundamental a investigação quanto à identificação da prevalência e avaliação do perfil de sensibilidade de amostras em profissionais da área de saúde que prestam cuidados direto ao paciente, particularmente os profissionais da Enfermagem, visando assegurar impacto positivo na assistência, bem como desenvolver o conhecimento sobre grupos de alto risco (MOREIRA et al., 2013; MOURA et al., 2011; OLSEN et al., 2013). Diante da magnitude do quadro apresentado, o presente trabalho buscou avaliar a prevalência de Staphylococcus aureus resistente à meticilina (MRSA) nas mãos e mucosa nasal de profissionais de Enfermagem de um hospital da cidade de Cascavel, Paraná, Brasil.

\section{METODOLOGIA}

\subsection{POPULAÇÃO DE ESTUDO}

O presente estudo foi realizado com profissionais de Enfermagem de um hospital da cidade de Cascavel, Paraná, durante o período de agosto a outubro de 2015, na unidade de terapia intensiva (UTI), centro cirúrgico, unidades de internamento particular/convênio e unidades de internamento do SUS do respectivo hospital. O projeto foi aprovado pelo Comitê de Ética em Pesquisa com Seres Humanos da Faculdade Assis Gurgacz (FAG), 
sob o parecer número 1.174 .353 de 20 de agosto de 2015. Participaram deste estudo enfermeiros e técnicos de Enfermagem que trabalham junto ao paciente, sendo a abordagem dos funcionários feita de forma aleatória e voluntária a partir da assinatura do Termo de Consentimento Livre e Esclarecido (TCLE), com intuito de coletar as amostras das mãos e da mucosa nasal destes profissionais e posterior aplicação do questionário. $\mathrm{O}$ questionário aplicado aos profissionais contou com informações sobre sexo, idade, categoria profissional, unidade de trabalho e uso de antibioticoterapia recente ou atual. A cada voluntário da pesquisa, foi atribuído um código, com a finalidade de manter o anonimato de cada participante.

O estudo contou com uma população amostral de 50 indivíduos, totalizando 200 amostras, com intervalo de coleta de trinta dias. Os profissionais que não completaram duas coletas e que realizaram o uso de antibioticoterapia nos últimos sete dias foram excluídos do estudo.

2.2 COLETA E ANÁLISE LABORATORIAL MICROBIOLÓGICA

As amostras foram coletadas dos profissionais durante o desenvolvimento de suas atividades junto aos pacientes, através de swab estéril. O material foi coletado pelo pesquisador, através da realização de movimentos circulares e delicados na fossa nasal anterior e nas mãos de cada profissional. Após cada coleta, as amostras foram encaminhadas imediatamente ao laboratório de microbiologia da Faculdade Assis Gurgacz (FAG), em tubos estéreis, contendo solução salina também estéril, conforme metodologia preconizada por Pereira e Cunha (2009).

Cada amostra foi inicialmente inoculada por técnica de esgotamento em placa em meio de cultura manitol salgado 7,5\%. As colônias sugestivas de $S$. aureus foram submetidas à coloração de Gram e a testes bioquímicos: Catalase, DNase, Coagulase, para confirmação da presença se Staphylococcus aureus.

Após identificação da presença de $S$. aureus nas amostras, as colônias identificadas com o agente foram novamente semeadas em ágar manitol e mantidas a $37^{\circ} \mathrm{C}$ por 24 horas a fim de se isolar o microrganismo. A partir do isolamento do mesmo, foi realizada uma suspensão da colônia na escala 0,5 de MacFarland e efetuado um inoculo em ágar Muller Hinton, a fim de se realizar o teste de sensibilidade por disco difusão (Kirby-Bauer) de acordo com as recomendações do CLSI (Clinical and Laboratory Standards Institute), utilizando-se o disco de oxacilina 1 ug e o de cefoxitina 30 ug (CLSI, 2013). Após incubação entre $37-38{ }^{\circ} \mathrm{C}$ por 16 a 18 horas, os halos foram medidos e comparados segundo critérios também estabelecidos pela CLSI, na qual foram considerados MRSA os $S$. aureus que formaram halo menor ou igual $11 \mathrm{~mm}$ com o disco da oxacilina ou os que apresentaram halo menor ou igual $21 \mathrm{~mm}$ com o disco da cefoxitina (CLSI, 2013).

Em todo o processo de análise microbiológica, foram utilizados controles positivos e negativos.

\subsection{ANÁLISE ESTATÍSTICA}

Os dados coletados foram armazenados em Microsoft Excel ${ }^{\circledR}$ e para análise e estudo sobre a associação entre variáveis adotou-se o teste de Qui Quadrado de Pearson (teste $\mathbf{x}^{2}$ ) em nível de significância de 5,0\%, com resultados considerados estatisticamente significativos para $p \leq 0,05$. A análise estatística foi realizada através do software STATA ${ }^{\circledR}$, versão 9.1.

\section{RESULTADOS}

Durante agosto a outubro de 2015, foram coletadas em dois momentos amostras da região nasal e de mãos de 50 profissionais de Enfermagem, com um intervalo de trinta dias entre as coletas, respeitando o preenchimento dos critérios de inclusão do presente estudo. No final do estudo, obteve-se 200 amostras biológicas.

Desta forma, dos 50 profissionais de Enfermagem que participaram da pesquisa, 46 (92\%) eram do gênero feminino e 4 (8\%) do masculino, ambos com faixa etária entre 18 a 50 anos. A população foi composta por 44 (88\%) técnicos de Enfermagem e 6 (12\%) enfermeiros, sendo que 24 (48\%) trabalhavam em sistema integral, 16 (32\%) 
no período da manhã e 10 (20\%) no período noturno. Quanto ao local de trabalho, 9 (18\%) trabalhavam na Unidade de Terapia Intensiva (UTI), 11 (22\%) no centro cirúrgico, 25 (50\%) nos leitos particular/convênio e 5 (10\%) nos leitos do SUS.

Verificou-se que dentre os indivíduos que participaram da presente pesquisa, a prevalência geral foi de 56\% (28/50) de colonização. Enquanto 23 (82,15\%) dos colonizados apresentaram colonização exclusivamente na cavidade nasal, 5 (17,85\%) apresentaram colonização em ambos os sítios anatômicos investigados (mãos e cavidade nasal) e nenhum apresentou colonização exclusiva nas mãos.
A análise por meio de estatística descritiva demonstrou que entre os 28 profissionais que apresentaram positividade para a presença de $S$. aureus, $89,29 \%$ (25/28) eram do gênero feminino e $10,71 \%$ (3/28) do gênero masculino. Quanto à faixa etária predominante dos profissionais colonizados por $S$. aureus, verificou-se que apresentaram entre 26 e 36 anos 13/28 (46,42\%). A grande maioria dos profissionais colonizados tinha como local de trabalho leitos particular/convênio (42,85\%), sob período integral (42,85\%). Entre os colonizados, $96,42 \%$ (27/28) eram técnicos em Enfermagem, enquanto 3,57\% (1/28) enfermeiros. As características gerais da equipe de Enfermagem encontram-se apresentadas na Tabela 1.

Tabela 1. Perfil epidemiológico e fatores de risco para colonização por Staphylococcus aureus em profissionais de Enfermagem de um hospital da cidade de Cascavel (PR), 2015

\begin{tabular}{|c|c|c|c|c|c|c|c|c|}
\hline Variáveis & $\begin{array}{c}\text { Colonizados } \\
\text { (n) }\end{array}$ & $\%$ & $\begin{array}{c}\text { Não } \\
\text { Colonizados } \\
\text { (n) }\end{array}$ & $\%$ & $\begin{array}{c}\text { Total } \\
\text { (n) }\end{array}$ & $\%$ & $\chi^{2}$ & OR (IC95\%) \\
\hline \multicolumn{9}{|l|}{ Gênero } \\
\hline Masculino & 3 & 10,71 & 1 & 4,54 & 4 & 8 & & 1 \\
\hline Feminino & 25 & 89,29 & 21 & 95,45 & 46 & 92 & 0,62 & $0,62(0,2327,10)$ \\
\hline \multicolumn{9}{|c|}{ Faixa etária (anos) } \\
\hline 18 a 25 & 5 & 17,85 & 3 & 13,63 & 8 & 16 & & 1 \\
\hline 26 a 36 & 13 & 46,42 & 9 & 40,90 & 22 & 44 & 0,03 & $0,87(0,16-4,71)$ \\
\hline 37 a 50 & 10 & 42,85 & 10 & 45,45 & 20 & 40 & 0,34 & $1,44(0,41-5,00$ \\
\hline \multicolumn{9}{|c|}{ Turno de trabalho } \\
\hline Manhã & 11 & 39,28 & 5 & 22,72 & 16 & 32 & & 1 \\
\hline Noturno & 5 & 17,87 & 5 & 22,72 & 10 & 20 & 0,88 & $2,2(0,41-11,94)$ \\
\hline Integral & 12 & 42,85 & 12 & 54,54 & 24 & 48 & 1,35 & $2,2(0,56-8,63)$ \\
\hline \multicolumn{9}{|c|}{ Categoria profissional } \\
\hline $\begin{array}{l}\text { Técnico em } \\
\text { Enfermagem }\end{array}$ & 27 & 96,42 & 17 & 77,27 & 44 & 88 & & 1 \\
\hline Enfermeiro & 1 & 3,57 & 5 & 22,72 & 6 & 12 & 4,2 & $7,94(0,75-83,84)$ \\
\hline \multicolumn{9}{|c|}{ Unidade de trabalho } \\
\hline UTI & 5 & 17,85 & 4 & 18,18 & 9 & 18 & 0,15 & $0,74(0,16-3,5)$ \\
\hline UIPC & 12 & 42,85 & 13 & 59,09 & 25 & 50 & & 1 \\
\hline UI-SUS & 3 & 10,71 & 2 & 9,09 & 5 & 10 & 0,23 & $0,62(0,08-4,52)$ \\
\hline Centro cirúrgico & 8 & 28,57 & 3 & 13,63 & 11 & 22 & 1,84 & $0,35(0,07-1,73)$ \\
\hline
\end{tabular}

OR: Odds Ratio; IC: Intervalo de Confiança; 1-Grupo de Referência; * $p<0,05$ : Estatisticamente significativo; UIPC - Unidade de internamento particular/convênio; UI-SUS - Unidade de internamento SUS

Fonte: Dados da pesquisa. 
Após a identificação de $S$. aureus dentre os profissionais de Enfermagem, as amostras desses profissionais foram submetidas ao teste de sensibilidade antimicrobiana frente à meticilina. Esta revelou que 8\% (4/50) apresentaram Staphylococcus resistente à meticilina (MRSA), sendo que os demais profissionais apresentaram Staphylococcus aureus sensível à meticilina (MSSA).

Dentre os aspectos demográficosdos portadores de MRSA analisados, a prevalência de indivíduos do gênero feminino foi de $75,00 \%$ (3/4), enquanto do gênero masculino foi de $25,00 \%(1 / 4)$. Quanto à faixa etária dos portadores de MRSA, 25,00\% apresentaram entre 18 a 25 anos e $50 \%$ entre 26 a 36 anos.

Em relação ao local de trabalho dos profissionais colonizados por MRSA 25,00\% (1/4) trabalhavam durante o turno da manhã e 25,00\% (1/4) no período noturno. A prevalência evidenciada foi durante o período integral com $50 \%(2 / 4)$.

Dentre os profissionais colonizados por MRSA, 100,00\% (4/4) da categoria profissional técnicos em Enfermagem demonstraram ser resistentes ao teste ao antimicrobiano, entretanto a análise estatística demonstrou que profissionais enfermeiros, correlacionavam-se com o desfecho de colonização. Verificou-se que a maioria dos funcionários colonizados por MRSA trabalham na UTI (50\%), entretanto não foi evidenciado nenhum profissional que trabalha no centro cirúrgico.

As características epidemiológicas dos indivíduos classificados segundo a colonização por MSSA e MRSA, encontram-se apresentadas na Tabela 2.

Tabela 2. Caracterização demográfica da equipe de Enfermagem que atua em um hospital da cidade de Cascavel (PR), segundo colonização S. aureus MSSA e MRSA. Cascavel (PR), 2015

\begin{tabular}{|c|c|c|c|c|c|c|c|c|}
\hline Variáveis & MSSA (n) & $\%$ & MRSA (n) & $\%$ & $\begin{array}{c}\text { Total } \\
\text { (n) }\end{array}$ & $\chi^{2}$ & OR (IC95\%) & $P$ \\
\hline \multicolumn{9}{|l|}{ Gênero } \\
\hline Masculino & 2 & 8,33 & 1 & 25,00 & 4 & & & \\
\hline Feminino & 22 & 91,67 & 3 & 75,00 & 24 & 0,96 & $0,27(0,02-4,41)$ & 0,33 \\
\hline \multicolumn{9}{|c|}{ Faixa etária (anos) } \\
\hline 18 a 25 & 4 & 16,67 & 1 & 25,00 & 5 & & 1 & \\
\hline 26 a 36 & 11 & 45,83 & 2 & 50,00 & 13 & 0,05 & $1,38(0,09-21,30)$ & 0,82 \\
\hline 37 a 50 & 9 & 37,50 & 1 & 25,00 & 10 & 0,60 & $2,25(0,1-52,40)$ & 0,60 \\
\hline \multicolumn{9}{|c|}{ Turno de trabalho } \\
\hline Manhã & 10 & 41,67 & 1 & 25,00 & 11 & & 1 & \\
\hline Noturno & 4 & 16,67 & 1 & 25,00 & 5 & 0,55 & $0,40(0,02-9,24)$ & 0,55 \\
\hline Integral & 10 & 41,67 & 2 & 50,00 & 12 & 0,60 & $0,50(0,04-6,94)$ & 0,28 \\
\hline \multicolumn{9}{|c|}{ Categoria profissional } \\
\hline $\begin{array}{l}\text { Técnico em } \\
\text { Enfermagem }\end{array}$ & 23 & 95,83 & 4 & 100,00 & 27 & 0,68 & $\#$ & 0,68 \\
\hline Enfermeiro & 1 & 4,17 & 0 & 0,00 & 1 & & 1 & \\
\hline \multicolumn{9}{|c|}{ Unidade de trabalho } \\
\hline UTI & 3 & 12,50 & 2 & 50,00 & 5 & & 1 & \\
\hline UIPC & 11 & 45,83 & 1 & 25,00 & 12 & 2,29 & $7,33(0,36-151,43)$ & 0,13 \\
\hline UI-SUS & 3 & 8,33 & 1 & 25,00 & 4 & 0,03 & $1,33(0,05-32,96)$ & 0,86 \\
\hline Centro cirúrgico & 8 & 33,33 & 0 & 0,00 & 8 & 3,49 & \# & 0,06 \\
\hline
\end{tabular}

OR: Odds Ratio; IC: Intervalo de Confiança; 1-Grupo de Referência; * $p<0,05$ : Estatisticamente significativo; \# - Não foi possível calcular o Odds Ratio; UIPC - Unidade de internamento particular/convênio; UI-SUS- Unidade de internamento SUS.

Fonte: Dados da pesquisa. 


\section{DISCUSSÃO}

No presente estudo, a taxa de presença quanto à colonização por Staphylococcus aureus foi de 56\%. Ao analisar a suscetibilidade antimicrobiana constatou-se uma prevalência de 92\% (46/50) de MSSA e 8\% (4/50) de MRSA. Estes dados levam a ressaltar a importância dos estudos de investigação da prevalência de Staphylococcus aureus e o seu perfil de resistência dentre os profissionais da área da saúde.

Analisando-se a taxa da prevalência de colonização por S. aureus dentre os profissionais de Enfermagem, nota-se que este valor encontra-se de acordo com os dados reportados na literatura quanto à presença dessa bactéria entre membros da equipe médica no Brasil. Leite (2008) demonstrou prevalência entre $33,3 \%$ e $85,7 \%$, e uma prevalência geral de $62,5 \%$ (10/16), foi evidenciada por Palos et al. (2009), entre profissionais pesquisados de um hospital de Goiânia, Goiás. Entretanto uma menor incidência $(24,4 \%)$, foi demonstrada por Kozesinski et al. (2014) entre profissionais de um hospital em Curitiba (PR).

Estudos sobre a ocorrência de Staphylococcus aureus em outros países também foram realizados. Em um trabalho efetuado com enfermeiros e residentes de Enfermagem nos Estados Unidos, verificou-se ocorrência de colonização por $S$. aureus em $62 \%$, demonstrando alta prevalência de colonização entre estes profissionais (MODY et al., 2008). Embora exista uma diversidade de resultados publicados na literatura, considerou-se elevada a prevalência de $S$. aureus no presente estudo (56\%).

A frequência de Staphylococcus aureus meticilina resistente (MRSA), observada neste estudo (8\%), corrobora com um inquérito realizado por Faria et al. (2011), na qual uma investigação sobre a prevalência de MRSA entre estudantes de Enfermagem igualmente demonstrou colonização em $8,8 \%$. Já uma investigação na Holanda apontou uma prevalência menor que 5\% dentre portadores colonizados por MRSA (BLOK et al., 2003) enquanto em Berlim, Alemanha, uma taxa de 1,6\% foi identificada (KAMPF et al., 2003). Outro estudo também na Alemanha identificou $36,4 \%$ de $S$. aureus dentre enfermeiros (KAMINSKI et al., 2007).

A prevalência de MRSA encontrada no presente estudo é considerada relevante quando se trata de indivíduos assintomáticos, uma vez que a oxacilina é um importante marcador de resistência para outros antimicrobianos, como os aminoglicosídeos, macrolídeos, cloranfenicol, tetraciclina e fluoroquinolonas (MANDELL et al., 2005).

A prevalência da colonização de $S$. aureus exclusivamente no nariz $(82,14 \%)$ e a colonização simultaneamente no nariz e mãos $(17,85 \%)$ verificados no presente estudo estão em concordância com relatos apresentados na literatura, nas quais as narinas constituem o principal sítio de colonização do $S$. aureus (PRATES et al., 2010). Compreende-se que a partir do estabelecimento da colonização nasal, o indivíduo contamina as próprias mãos e passa a ser carreador da bactéria (LIMA et al., 2015). Diante desses achados, é indispensável que se realizem sistematicamente palestras informativas e de conscientização, enfatizando a importância da lavagem correta das mãos no controle da infecção adquirida na comunidade e nos hospitais (RIBEIRO et al., 2014).

Diversos estudos têm mostrado que colaboradores de hospitais são responsáveis pela transmissão de Staphylococcus aureus no contexto hospitalar. Esse evento deve-se devido aos profissionais serem conhecidos por conter as maiores taxas de estirpes de resistência nas narinas que a população geral (BRIAN et al., 2008).

Dentre as variáveis investigadas nesse estudo, sexo, idade, turno de trabalho e unidade de trabalho, não foi verificada relação estatística com a colonização pelo $S$. aureus $(p>0,05)$.

A taxa geral de colonização por $S$. aureus dentre os profissionais de Enfermagem foi maior no gênero feminino $(82,14 \%)$, em relação ao sexo masculino (10,71\%). Esse dado se assemelha aos achados por Carvalho et al. (2009), Campiotto et al. (2010) e Moura et al. (2011), nas quais esses autores também evidenciaram prevalência no gênero feminino quanto à colonização de S. aureus em profissionais de Enfermagem.

Trabalhos em serviços de saúde são fatores de risco ambiental para o carreamento de S. aureus para outras pessoas, devido ao contato entre enfermeiros e auxiliares de Enfermagem com doentes e idosos em unidades de assistência à saúde, uma vez que a transmissão dessa bactéria de pacientes à equipe de saúde também pode ocorrer (OLSEN et al., 2013; CÁCERES, 2011). 
Em relação aos colaboradores colonizados por MSSA, a maior parte era do gênero feminino, assim como o visualizado com os portadores de MRSA. Esse estudo difere dos estudos de Freire et al. (2013), Sangvik et al. (2011) e Rongpharpi etal. (2013), em que a predominância da colonização por S. aureus foi em indivíduos do gênero masculino. Entretanto, o resultado do presente estudo se assemelha ao verificado por Elahi et al. (2013) tanto para os portadores de MSSA como de MRSA.

Em relação à faixa etária, verificou-se que a maioria dos portadores possuía idade entre 26 e 36 anos. Esse dado se assemelha aos dados encontrados por Campiotto et al. (2010), onde a maior representatividade das culturas consistia de portadores com idade entre 20 e 40 anos (89,7\%). Em um estudo realizado em Santo André, São Paulo, realizado entre os anos de 2006 a 2008, foi demonstrado que a idade que prevaleceu entre os profissionais colonizados foi entre 19 e 39 anos $(69,7 \%)$ (CARVALHO et al., 2009).

Avaliando-se a condição de colonização, por unidade de trabalho, observou-se que as unidades com maior percentual de trabalhadores não colonizados foi a unidade de internamento do SUS (10,71\%), enquanto a unidade com mais colonizados foi a unidade de internamento particular (42,85\%). Os setores com maior prevalência de colaboradores carreadores de MSSA foi a unidade de internamento particular/convênio (45,83\%). Destaca-se que entre as unidades com maior percentual de colonizados por MRSA a Unidade de Terapia Intensiva (UTI) (50\%), enquanto não foram identificados portadores de MRSA na unidade de centro cirúrgico.

O quadro com maior percentual de MRSA pode ser justificado por se tratar de amostras de funcionários de UTI, setor no qual se concentram os maiores fatores de risco para aquisição de microrganismos multirresistentes, dentre os quais a gravidade de casos clínicos, tempo de internação, uso de procedimentos invasivos e antibioticoterapia indiscriminada (MOURA et al., 2011; MOREIRA et al., 2013).

A prevalência de MRSA chama a atenção em alguns setores específicos, uma vez que um único carreador de MRSA pode se tornar responsável pela transmissão deste microrganismo aos seus pacientes, para tal situação considera-se relevante a descolonização do profissional colonizado e culturas de vigilância da equipe (MOURA et al., 2011). Patel et al. (2008) constataram em seu estudo que entre os pacientes de UTI, a colonização por MRSA foi associada à infecção subsequente e risco de morte, concluindo que estudo de vigilância de MRSA pode identificar os indivíduos colonizados para desfechos adversos e melhorar os programas de prevenção.

Para Moura et al. (2011) menores incidências entre profissionais de Enfermagem presume-se ao fato de que quando os trabalhadores têm ciência da condição de portador de seus pacientes, isso os leva a tomarem mais cuidado ao manuseá-los, ou seja, a percepção de risco de contaminação leva o trabalhador a se proteger melhor.

Esse estudo demonstra que os profissionais que trabalham no período integral apresentam maior índice de colonização por $S$. aureus (42,85\%), bem como por MSSA $(41,67)$ e MRSA (50\%). Esta ocorrência pode ser explicada por Cruz (2008), na qual a longa jornada de trabalho pode favorecer erros e desatenção, assim como maior tempo de exposição ao ambiente insalubre.

Analisando-se as categorias profissionais em estudo, enfermeiros apresentaram relação estatística com a colonização pelo $S$. aureus $(p<0,05)$, contudo profissionais técnicos em Enfermagem demonstraram ter maior proporção quanto à incidência da colonização pela bactéria, bem como por MSSAe MRSA. Nos estudos de Palos (2009), Cruz (2008) e Campiotto et al. (2010) também foi verificada maior incidência entre colaboradores técnicos em Enfermagem e menor prevalência entre os colaboradores enfermeiros.

Dulon et al. (2014) asseguram que o risco do pessoal de Enfermagem ser colonizado com MRSA é quase duas vezes maior do que para o pessoal médico e três vezes maior do que para outros profissionais de saúde. Esse fato é assegurado tendo em vista que o transporte nasal de MRSA em profissionais de Enfermagem é mais elevado do que em outros profissionais da saúde, tendo em vista que o profissional de Enfermagem desempenha suas atividades cotidianas diretamente relacionadas ao paciente (OLSEN et al., 2013).

Rongpharpi et al. (2013) diante do quadro da colonização por $S$. aureus assinalam que dentre os fatores de prevenção de infecções relacionadas à assistência a saúde - IRAS, está o cumprimento de medidas sanitárias e 
orientação do uso de antimicrobianos por profissionais de saúde. Dentre as medidas apontadas pelo autor, incluem procedimentos simples, como lavar as mãos antes e depois do exame do paciente, o uso de aventais estéreis e máscaras nas enfermarias pós-operatórias, a consciência durante o exame de pacientes imunocomprometidos, evitando tocar o nariz durante o trabalho, medidas como estas são aliadas às ações educativas, treinamentos sobre a manutenção da higiene e controle de infecção podem reduzir consideravelmente a taxa de transmissão da doença.

O monitoramento da prevalência de transporte nasal, bem como a transmissão de estirpes resistentes a múltiplas drogas (MDR) de Staphylococcus aureus de origem hospitalar é essencial. Devido a essas cepas quando transmitidas a partir de pessoal hospitalar a pacientes com estados imunitários enfraquecidos ou já embutido dispositivos médicos, podem limitar as opções de tratamento (EYOH et al., 2014).

Investigações para desvendar a dinâmica de fatores de virulência e as complexidades de estirpes como MSSA e MRSA devem ser realizadas. Sobretudo, a prevenção de colonização por MRSA na equipe de Enfermagem deve ser investigada, já que este grupo profissional parece experimentar o maior risco para a colonização por MRSA (RICHARD et al., 2012; DULON et al., 2014).

Além dos instrumentos preventivos da colonização por $S$. aureus, na atualidade, as ferramentas de epidemiologia molecular também permitiram grande avanço na investigação dos processos infecciosos, principalmente na elucidação de surtos epidêmicos de infecções, pois permitem identificar e caracterizar com segurança os agentes etiológicos, bem como possibilita determinar origens clonais dos isolados (SILVA et al., 2012).

Embora existam controvérsias na literatura sobre o papel dos profissionais de saúde na transmissão de Staphylococcus aureus e daquelas cepas resistentes à meticilina (MRSA), profissionais que apresentam transitoriamente ou persistentemente o microrganismo são responsáveis pelo transporte aos pacientes e a infecção subsequente. Sendo assim, estudos sobre a identificação de portadores assintomáticos de $S$. aureus em profissionais de saúde são de grande importância na epidemiologia e patogênese da doença.

\section{CONCLUSÃO}

Entre as 200 culturas de mãos e vestíbulos nasais dos colaboradores de Enfermagem de um hospital da cidade de Cascavel (PR) foi encontrada prevalência de 56\% de profissionais da saúde colonizados por S. aureus, dos quais $92 \%$ eram colonizados por MSSA e $8 \%$ por MRSA.

A partir dos dados apresentados ressalta-se a importância da pesquisa de Staphylococcus aureus dentro da equipe de Enfermagem que atua a nível hospitalar, devido aos mesmos estarem diretamente inseridos em grupos vulneráveis à presença de microrganismos de diferentes estirpes. Durante a prática profissional a equipe de Enfermagem desenvolve suas atividades junto ao paciente, podendo ser disseminadores de patógenos. Este trabalho aponta ainda para a necessidade de estudos epidemiológicos de vigilância quanto à disseminação de microrganismos em nível hospitalar.

\section{REFERÊNCIAS}

ATIQUE, T. S. C.; LIMA, T. A. M.; SOUZA, V. A.; PACHECO, P. F. C.; FURINI, A. A. C. Sensibilidade à meticilina/ oxacilina de Staphylococcus aureus isolados da mucosa nasal de alunos do Centro Universitário de Rio Preto. Rev. Bras. Farm., v. 93, n. 3, p. 347-352, 2012.

BALDUINO, L. G. Análise de portadores assintomáticos de Staphylococcus aureus no Hospital Universitário de Brasília. 2008. 102 f. Dissertação (Mestrado Patologia Molecular) - Universidade de Brasília, Faculdade de Medicina da, Brasília, DF.

BASTOS, V. M. P.; NORBERG, A. N.; OLIVEIRA, J. T. M.; SANCHES, F. G.; BARRETO JUNIOR, O. S.; SERRA-FREIRE, N. M. Comparação da incidência, da prevalência da colonização, e da resistência de Staphylococcus aureus em diferentes populações humanas. Rev UNIABEU, Belford Roxo, v. 6, n. 13, p. 28-40, 2013.

BLOK, H. E.; TROELSTRA, A.; KAMP-HOPMANS, T. E.; GIGENGACK-BAARS, A. C.; VANDENBROUCKE-GRAULS, C. M.; WEERSINK, A. J. et al. Role of healthcare orkers in 
outbreaks of methicillin-resistant Staphylococcus aureus: a 10-year evaluation from a Dutch university hospital. Infect Control Hosp Epidemiol., v. 24, n. 9, p. 679-685, 2003.

BRASIL. Agência Nacional de Vigilância Sanitária. Ministério da Saúde. Microbiologia clínica para o controle de infecção relacionada à assistência à saúde. Módulo 5: Tecnologias em Serviços de Saúde: descrição dos meios de cultura empregados nos exames microbiológicos. Brasília: Anvisa, 2013.

BRIAN, P. S.; ELIOT, H. C.; KAVEH, I.; DONALD, M. Y. Prevalence of Staphylococcus aureus Nasal Colonization in Emergency Department Personnel. Ann Emerg Med., v. 52, n. 5, p. 529-533, 2008.

CÁCERES, M. Frecuencia de portadores nasales de Staphylococcus aureus resistente a meticilina em personal de salud de hospitais de Nicaragua. Rer Panam Salud Publica, v. 30, n. 6, p. 610-614, 2011.

CAMPIOTTO, L. G.; FACCIN, M. C.; PEREIRA, E. C. A.; YAMAGUCHI, M. U.; KEMMELMEIER, G. S. Staphylococcus aureus em profissionais da saúde: prevalência e perfil de resistência aos agentes antimicrobianos. In: MOSTRA INTERNA DE TRABALHOS DE INICIAÇÃO CIENTÍFICA, 5., 2010, Anais... Maringá: Diretoria de Pesquisa Cesumar, 2010.

CARVALHO, M. J.; PIMENTA, F. C. P.; HAYASHIDA, M.; GIR, E.; SILVA, A. M.; BARBOSA, C. P.; CANINI, S. R. M. S.; SANTIAGO, S. Prevalence of methicillin-resistant and methicillin - susceptible $S$. aureus in the saliva of health professional. Clinics, v. 64, n. 4, p. 295-302, 2009.

CASTRO, O. R.; FERRER, L. M. V.; RIVERA, E. A.; ARCO, M. M.; DONADO, R.; HEINS, G. V. V. Staphylococcus aureus meticilino resistente em niños escolares de Cartagena. Rev. Salud pública, v. 12, n. 3, p. 454-463, 2010.

CLINICAL LABORATORY STANDARDS INSTITUTE. Performance Standards for Antimicrobial Susceptibility Testing: Twenty-third Informational Supplement M100-S23. CLSI, Wayne, PA, USA, 2013.

CORREAL, J. C. D.; MARQUES, E. A.; GULHERME, W. L.; LEÃO, R. S.; DAMASCO, P. V. Infecções por Staphylococcus aureus: mudança do perfil epidemiológico no Hospital Universitário Pedro Ernesto. Revista HUPE, Rio de Janeiro, v. 12, n. 3, p. 31-46, 2013.

CRUZ, E. D. A. Staphylococcus aureus e Staphylococcus resistente à meticilina em trabalhadores de um hospital universitário: colonização e crenças em saúde. 2008. Tese (Doutorado) - Universidade de São Paulo, Escola de Enfermagem de Ribeirão Preto, SP.

DULON, M.; PETERS, C.; SCHABLON, A.; NIENHAUS, A. MRSA carriage among healthcare workers in nonoutbreak settings in Europe and the United States: a systematic review. BMC Infect Dis., v. 14, p. 363, 2014.

SAADAATIAN-ELAHI, M.; TRISTAN, A.; LAURENT, F.; RASIGADE, J. P.; BOUCHAIAT, C.; RANC, A. G.; LINA, G.; DAUWALDER, O.; ETIENNE, J.; BES, M.; VANDENESCH, F. Basic Rules of Hygiene Protect Health Care and Lab Workers from Nasal Colonization by Staphylococcus aureus: An International Cross-Sectional Study. PloS One, v. 8, n. 12, e82851, 2013.

EYO, A. B.; TOUKAM, M.; ATASHILI, J. FOKUNANG, C.; GONSU, H.; LYONGA, E. E. et al. Relationship between multiple drug resistance and biofilm formation in Staphylococcus aureus isolated from medical and nonmedical personnel in Yaounde, Cameroon. Pan Afr Med J., v. 17, p. 186, 2014.

FARIA, S. T.; PIEKARSKI, A. R.; TOGNIM, M. C. B.; BORELLI, S. D.; BEDENDO, J. Perfil fenotípico e genotípico de Staphylococcus aureus isolados de estudantes de enfermagem. Acta Paul Enferm., v. 24, n. 2, p. 213-218, 2011.

FELIX, C. C. P.; MIYADAHIRA, A. M. K. Avaliação da técnica de lavagem das mãos executada por alunos do Curso de Graduação em Enfermagem. Rev esc enferm. USP, v. 43, n. 1, 2009.

FREIRE, I. L. S.; MENEZES, L. C. C.; SOUSA, N. M. L.; ARAUJO, R. O.; VASCONSELOS, Q. L. D. A. Q.; TORRES, G. $\mathrm{V}$. Epidemiologia das infecções relacionadas à assistência à saúde em unidade de terapia intensiva pediátrica. Rev Bras Ciências da Saúde, v. 11, n. 35, 2013. 
KAMINSKI, A.; KAMMLER, J. M.; WICK, M. G.; KUTSCHALISSBERG, F. Transmission of methicillin-resistant Staphylococcus aureus among hospital staff in a German trauma centre: a problem without a current solution? J Bone Joint Surg Br., v. 89, n. 5, p. 642-645, 2007.

KAMPF, G.; ADENA, S.; RUDEN, H.; WEIST, K. Inducibility and potential role of MecA-gene-positive oxacillinsusceptible Staphylococcus aureus from colonized healthcare workers as a source for nosocomial infections. J Hosp Infect., v. 54, n. 2, p. 124-129, 2003.

KOZESINSKI, A. C. C.; SILVA, S. E. F. Prevalência de Staphylococcus aureus e sua variante resistente à meticilina e sua relação com local e tempo de trabalho em profissionais da saúde de Curitiba. In: CONGRESSO SUL BRASILEIRO DE INICIAÇÃO CIENTÍFICA \& PÓSGRADUAÇÃO, 3., 2014. Anais... Curitiba: PUC, 2014.

LEITE, G. B. Análise de portadores assintomáticos de Staphylococcus aureus no hospital universitário de Brasília. 2008. Dissertação (Mestrado em Patologia Molecular) - Universidade de Brasília, Faculdade de Medicina, Brasília.

LIMA, M. F. P.; BORGES, M. A. B.; PARENTE, R. S. P.; VICTÓRIAJUNIOR, R. C.; OLIVEIRA, M. E. Staphylococcus aureus e as infecções hospitalares: revisão de literatura. Rev UNINGÁ Revew, v. 21, n. 1, p. 32-39, 2015.

MASUNARI, A.; TAVARES, L. C. Estudos de QSAR-3D em derivados 5-nitro-2-tiofilidênicos com atividade frente a Staphylococcus aureus multi-resistente. Rev bras ciênc farm., v. 43, n. 2, p. 281-294, 2007.

MANDELL, G. L.; BENNETT, J. E.; DOLIN, R.; MANDELL, D. B. Principles and practice of infectious diseases. $6^{\text {th }}$ ed. Philadelphia: Churchill Livingstone, 2005.

MODY, L.; KAUFFMAN, C. A.; DONABEDIAN, S.; ZERVOS, M.; BRADLEY, S. F. Epidemiology of Staphylococcus aureus colonization in nursing home residents. Clin Infect Dis., v. 46, n. 6, p. 1368-1373, 2008.

MOREIRA, A. C. M. G.; SANTOS, R. R.; BEDENDO, J. Prevalência e perfil de sensibilade de Staphylococcus aureus em pacientes e equipe de enfermagem. Cienc Cuid Saude, v. 12, n. 3, p. 572-579, 2013.
MOURA, J. P.; PIMENTA, F. C.; HAYASHIDA, M.; CRUZ, E. D. A.; CANINI, S. R. S.; GIR, E. A colonização dos profissionais de enfermagem por Staphylococcus aureus. Rev. Latino-Am. Enfermagem, v. 19, n. 2, 2011.

OLIVEIRA, A. C.; SILVA, M. D. N.; GARBACCIO, J. L. Vestuário de profissionais de saúde como potenciais reservatórios de microrganismos: uma revisão integrativa. Texto Contexto Enferm., Florianópolis, v. 21, n. 3, p. 684-691, 2012.

OLSEN, K.; SANGVIK, M.; SIMONSEN, G. S.; SOLLID, J.; SUNDSJORD, A.; FURBERG, A. S. Prevalence and population structure of Staphylococcus aureus nasal carriage in healthcare workers in a general population. The Troms Staph and Skin Study. Epidemiology and Infection, v. 141, n. 1, p. 143-152, 2013.

PALOS, M. A. P.; SILVA, D. V. B.; GIR, E.; CANINI, S. R. M. S.; ANDERS, P. S.; LEÃO, L. S. N. O.; PIMENTA, F. C. Microbiota das mãos de mães de maternidade e de profissionais de saúde de uma maternidade de Goiânia. Rev Eletr Enf., v. 11, n. 3, p. 573-578, 2009.

PATEL, M.; WEINHEIMER, J. D.; WAITES, K. B.; BADDLEY, J. W. Active surveillance to determine the impact of methicillin-resistant Staphylococcus aureus colonization on patients in intensive care units of a Veterans Affairs Medical Center. Infect Control Hosp Epidemiol, v. 29, n. 6, p. 503-509, 2008.

PRATES, K. A.; TORRES, A. M.; GARCIA, L. B.; OGATTA, S. F. Y.; CARDOSO, C. L.; TOGNIM, M. C. B. Nasal carriage of methicillin-resistant Staphylococcus aureus in university students. Braz J Infect Dis., v. 14, n. 3, p. 316-318, 2010.

RIBEIRO, I. F.; SILVA, F. R. S.; SILVA, S. L.; RIBEIRO, M. M. N. P. R.; STOLP, A. M. V. Identificação de Staphylococcus aureus e Staphylococcus aureus resistente à meticilina em estudantes universitários. Rev Ciênc Farm Básica Apl., v. 35, n. 2, p. 301-304, 2014.

RICHARD, R. W.; MICHAEL, Z. D.; ROBERT, A. S. Current concepts on the virulence mechanisms of meticillinresistant Staphylococcus aureus. J Med Microbiol, v. 61, n. 9, p. 1179-1193, 2012. 
RONGPHARPI, S. C.; HAZARIKA, N. K.; KALITA, H. The Prevalence of Nasal Carriage of Staphylococcus Aureus Among Healthcare Workers at a Tertiary Care Hospital in Assam with Special Reference to MRSA. J. Clin Res Diagn., v. 7, n. 2, p. 257-260, 2013.

SANGVIK, M.; OLSSEN, R. S.; OLSEN, C.; SIMONSEN, G. S.; FURBERG, A. S. Age- and Gender-Associated Staphylococcus aureus spa Types Found among Nasal Carriers in a General Population: the Troms $\varnothing$ Staph and Skin Study. J. Clin. Microbiol., v. 49, n. 12, p. 4213-18, 2011.

SILVA, E. C. B. F.; SAMICO, T. M.; CARDOSO, R. R.; REBELO, M. A.; NETO, A. M. B.; MELO, F. L.; CATARINA, S. L.; ACA, I. S.; MACIEL, M. A. Colonização pelo Staphylococcus aureus em profissionais de enfermagem de um hospital escola de Pernambuco. Rev Esc Enferm USP, v. 46, n. 1, p. 132-1327, 2012.

SILVA, P. F.; PADOVEZE, M. C. Infecções relacionadas a serviços de saúde orientação para público em geral: conhecendo um pouco mais sobre infecção. Departamento de Enfermagem em Saúde Coletiva da Escola de Enfermagem da Universidade de São Paulo. São Paulo, 2012.

XAVIER, C. A. C.; OPORTO, C. F. O.; SILVA, M. P.; SILVEIRA, I. A. S.; ABRANTES, M. R. A. Prevalência de Staphylococcus aureus em manipuladores de alimentos das creches municipais da cidade de Natal/RN. Rev Bras Anal Clin., v. 39, n. 3, p. 165-168, 2007.

Recebido em: 04 de fevereiro de 2016 Aceito em: 29 de agosto de 2016 